
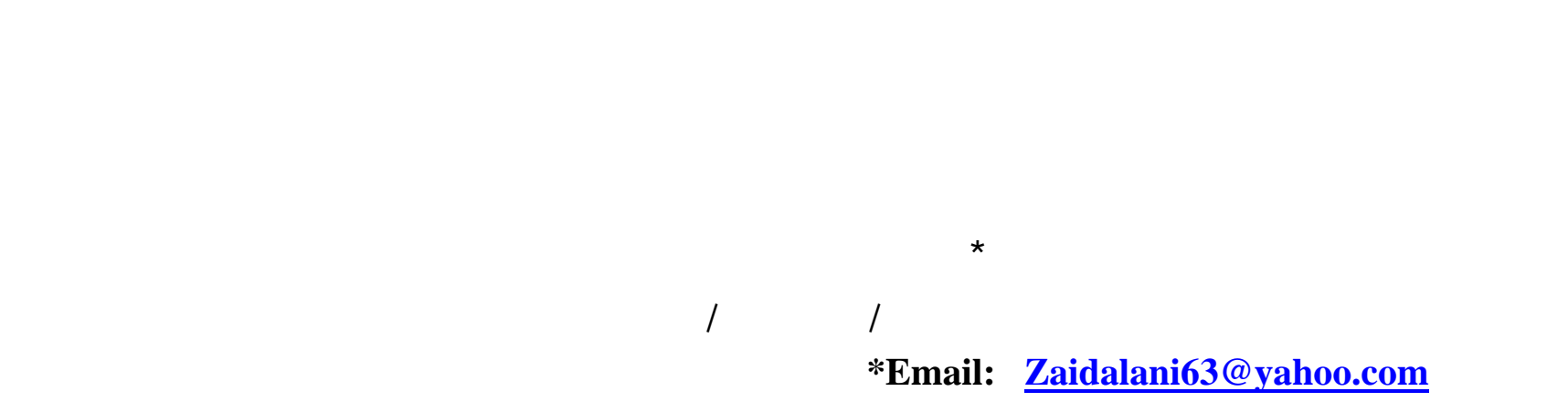

(2013/ 11 / 125 (منتلم 2013 / 2013 ؛

\title{
الملغص
}

لجريت هذه الدرلسة لمعرفة تأثير المستخلص المغلي القرفة بتركيز 2000 ملغم/كغم وزن الجم ف مي بع ـض المع اييبر الموية ومستوى هرمون اللبتن لإنت الأرافب النيوزلنية البيض للسليمة والمصابة بداء للسكري التجريبي والت مي تراوح ـات

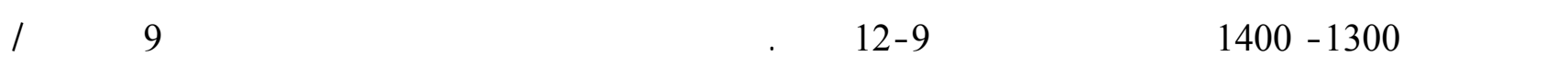
مجموعة، وكانت كالآتي:مجموعة للسيطرة، مجموعة القرفة، مجموعة الألوكسان ومجموعة القرفة مع الألوكسان معا. وتمت

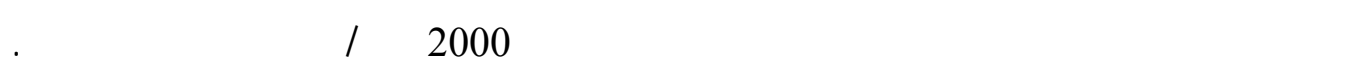

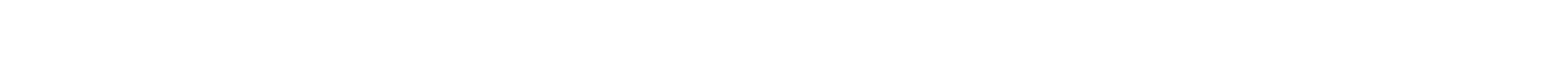
والمصابة بداء للسكر التجريبي، إذ رفعت معنويا أعداد كريت الدم الحمر وخلايا الهم البيض وتركي -ز الهيموكل ـوبين وحج م كريك الدم المرصوصة مقارنة مع مجموعتي الألوكسلن ومجموعة للسطرة وكذلك أدت المعلملة بمستخلص القرفة المغلي إلى اله

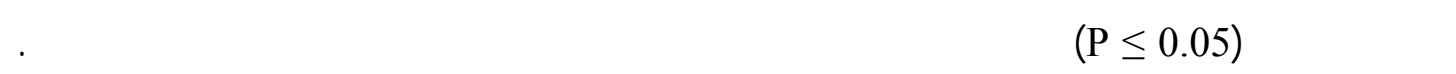
الهامل الدالة: القرفة، ألوكسان، هرمون اللبتين، الأرانب النيوزلندية البيض.

\section{Effect of Cinnamon Boiled Extract on Blood Picture and Leptin Hormone of Experimentally - Induced Diabetic White New Zealand Female Rabbits}

Zaid SH. Al- Ani

Muntaha M. Al-Kattan

Department of Biology / College of Science /University of Mosul

\section{ABSTRACT}

This study was conducted to investigate the effect of cinnamon boiled extract $(2000 \mathrm{mg} / \mathrm{kg}$ body weight) on some hematological parameters and Leptin hormone level in healthy and experimentally- induced diabetic white New Zealand female rabbits, it's weights ranged between 1300-1400 gm and aged 9-12 months. The rabbits were randomly divided into 4 groups, (9 rabbits / group) Control, Alloxan, Cinnamon and Cinnamon with Alloxan together group. The rabbits were dosaged and observed for four weeks.

The results showed that the cinnamon boiled extracts enhance the hematological parameter for the healthy and diabetic female rabbits. The treatment raised red blood corpuscles, total white blood cells, hemoglobin concentration and packed cell volume comparing with the Alloxan and Control group. Furthermore, the treatment with Cinnamon boiled extract increases the level of Leptin hormone comparing with the Alloxan and Control groups.

Keywords: Cinnamon, Alloxan, Leptin, White New Zealand rabbits. 


\section{اللقمة}

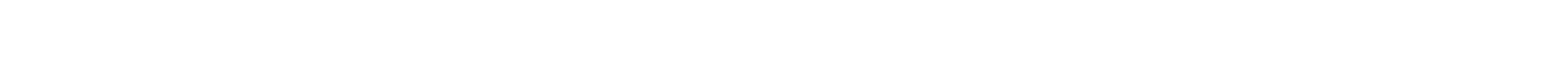

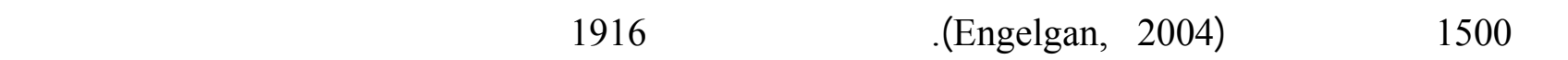

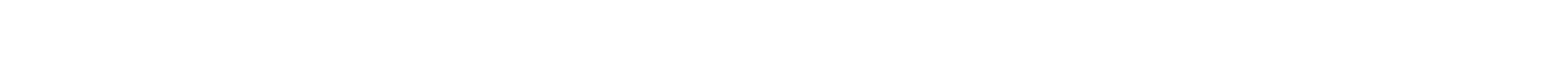

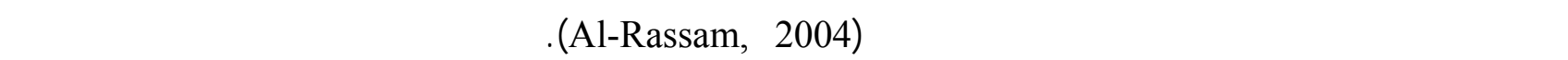

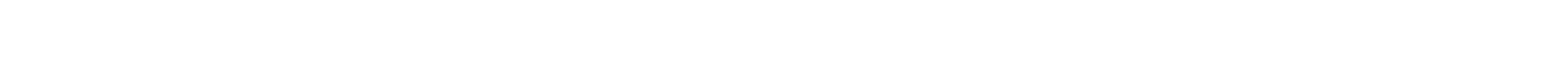

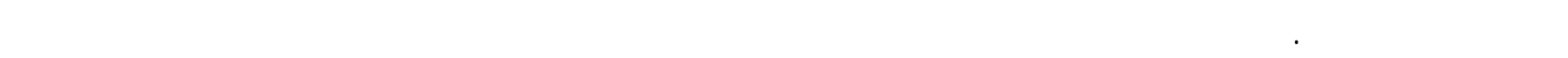
الطبية (Al-Rawi and Fetters , 2012). وعلى الرغم من الظور الكبير في مياين الكيمياء والصيدلة فقد عاد الإنسان من جديد إلى التداوي بالأعشب والنباتلا

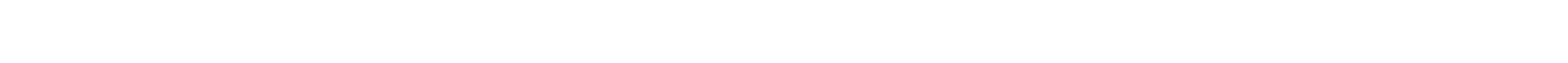

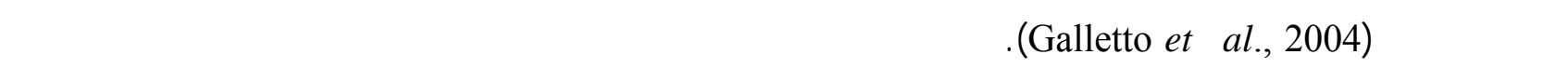
هو أحدمصادر العلاج الآمن. ومن النباتت التي تستخدم لقلبل مستوى المكر هو نبلت القرفة أو الداوسين Cinnamomum zylanicum

Classification of Plant

Kingdom: Plants

Subkingdom: Vascular Plants

Division: Flowering Plants

Class: Dicotyledons

Order: Laurales

Family: Lauraceae

Genus: Cinnamon

Species: Cassia

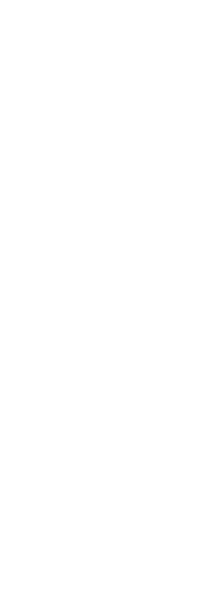

(Natural Resources Conservation Service, 2004)

أن الموطن الأصلي للداسيني هوسريلانكا، ولهذا اتخذ الداسيني اللسيلاني لسمه من موطنه الأصلي إذ ينمو في الغابلت

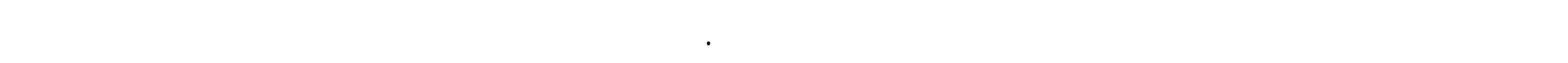
وعطري (تريز والفانز، 2003). تحتوي القرفة على للسينمالديهايد Cinnamaldehyde بذ سبة 55 - 80 \% وفيذ -ولات متع عدة اليوجينول Eugenol، ولسترات Esters وكميت قليلة من الكيتونات والكحولات و كما تحتوي على مواد صمغية واوك ـزالات

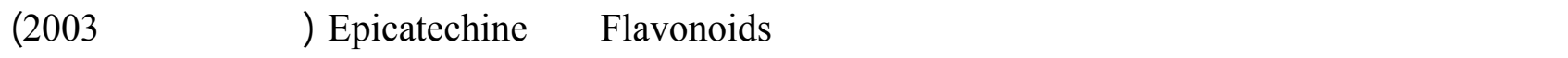
(Barnes et al., 2002)

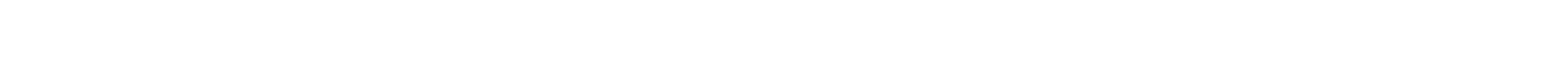
وللسقلن والأوراق والثمار إما علىشكل إضلفلت غذائية أومستخلصت مائية أوكحولية، وقد اهم البلحثن في مختلف أنحاء 
العالم بداء للسكر كونه من الأمراض الأثثرشيوعا في الوقت الحاضر و لغطورة مضاعفاته التي تؤدي إلى أم. _راض مه ددة للحيلة كإصابة القلب والكلية والأوعية الدموية والأعصلب وشبكية العن (Rajagopal and Sasikala , 2008). المركبلت الكيميائية للنبل: يحتوي الداسين على العديدمن المركبلت الكيميائية المختلفة و بنسب مختلفة و وت ششمل:

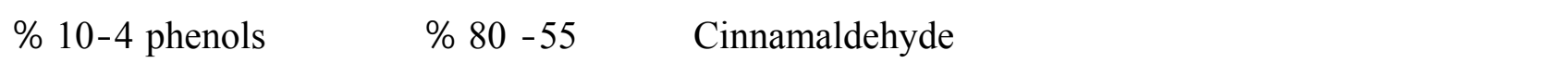
(مثل اليوجينول، مثل اليوجينول و اللسفرول)، هيدروكاربونات Hydrocarbons (مل بينين، فيلان درين وك الربوفيلايلين)،

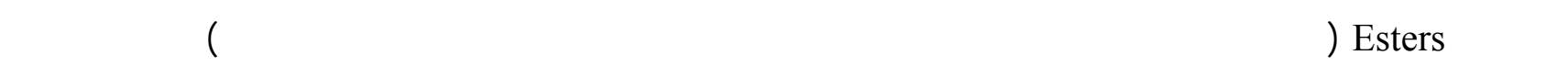
Cinnzeylanin ، للسيزيلانول Cinnzeylanol، كيتونات Ketones وكحولات Stcohols كما ويحوي على نشأ Starch، أليف Fucilage و Fers (تريز و الفانز، 2003)، و حلمض الدابس ين Cinnamonic acid (ال -ورع ولخ -رون،

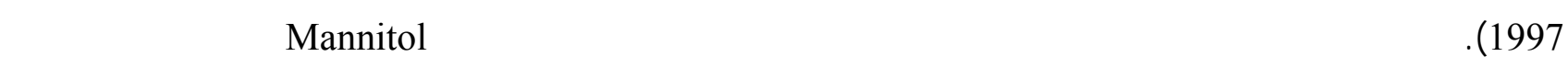

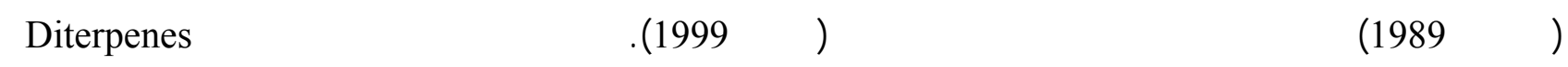
فلافونويدات، مواد لعابية وكفصية (1) (Anderson et al., 2004).

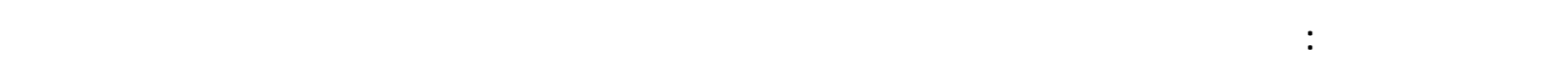
الأرافب النيوزلندية البيض المصابة بداء للسكر التجريبي.

\section{المولد طلراكق الهل}

الحيولنت : لمتخدم في هذه الدرلسة 36 من إنث الأرافب النيوزلنية البيض إذ م الحصول عليها من الأسواق المحلية

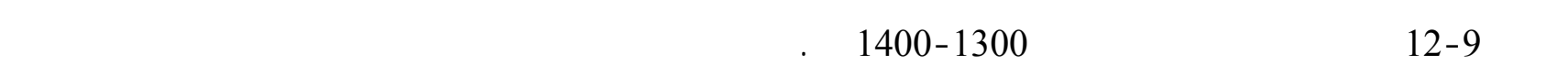

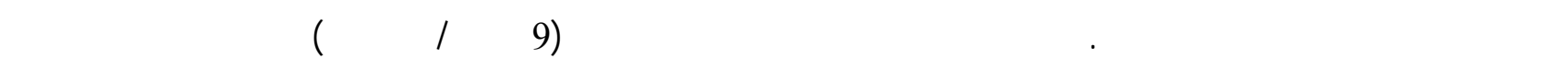

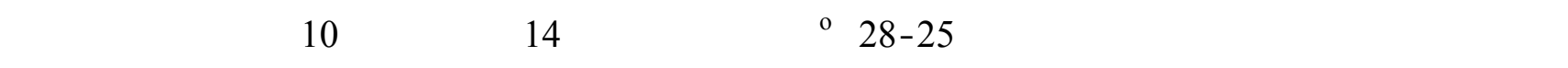

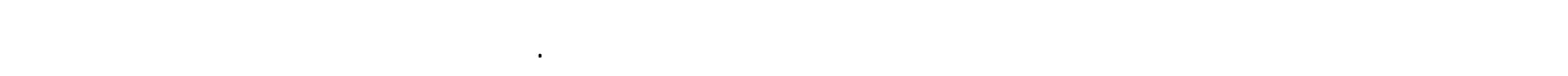
علف لخضر بنسبة 25\% من العليقة، وقمت مع الماء بشكل حر لجمبع الأرافب.

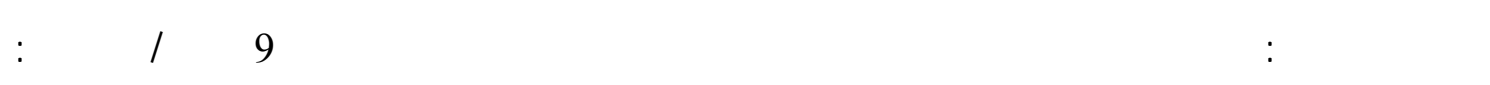
1 - مجموعة السيلرة: جرعت المل/كغم وزن جمه محلول فسلجي.

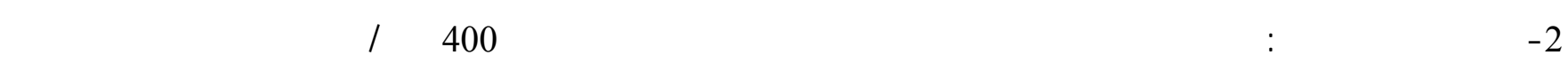

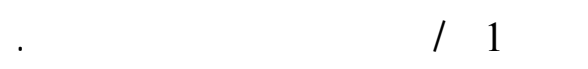
3 - مجموعة الفرفة: جرعت بمستخلص القرفة المغلي بتركيز 2000 ملغم الكغم وزن همبم.

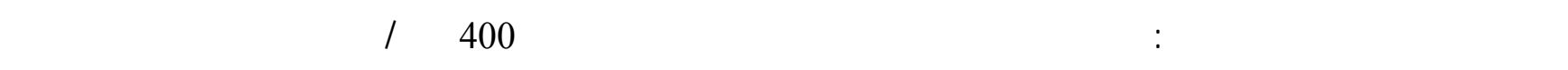

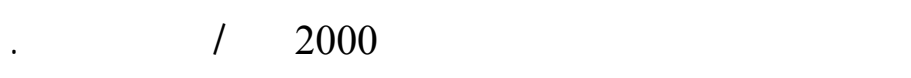


الجطل 1:مكونلت العلقة المستخدة في الدرلسة

\begin{tabular}{|c|c|c|c|}
\hline $\begin{array}{c}\text { \% البروتن الخلم \% } \\
\text { Crude protein }\end{array}$ & النسبة المئوبة \% & Ingredient & |المالة اللفية الأولية \\
\hline 7.50 & 47 & Wheat bran & نخالة حظة \\
\hline 3.60 & 38 & Local crushed barley & مجروششعير محلي \\
\hline 4.40 & 10 & Soybean meal & كسبة فول الصويا \\
\hline 1.0 & 2 & Protein concentration & مركز بروتيني (44\% بروتين) \\
\hline - & 1 & Limes binder & مسحوق حجر الكللس \\
\hline- & 1.5 & $\mathrm{NaCl}$ & ملح الطعلم \\
\hline- & 0.5 & Premix & خليط فيتلمينات ومعان \\
\hline$\% 16.5$ & $\% 100$ & Total & المجموع \\
\hline
\end{tabular}

النبل: لستخدم لحاء نبلت القرفة Cinnamomum zylanicum (اللهم اللشعبي الشائع: الدابسيني) إذ ت م الح صصول

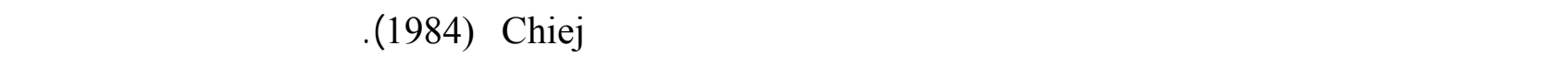

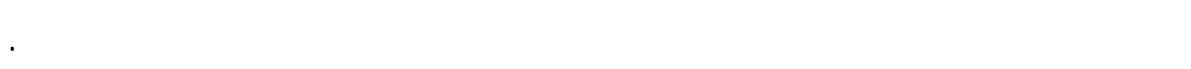

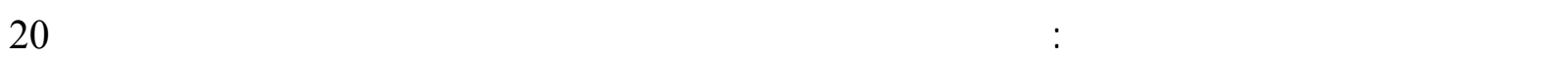

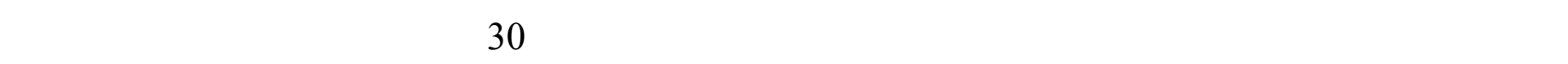

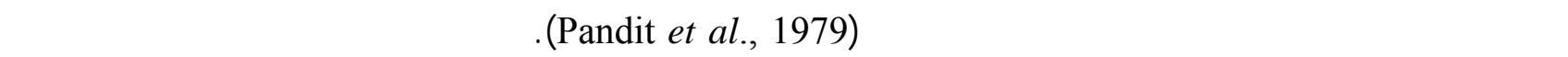

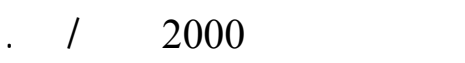

لمتحداث داء السكر النجريب Induction of Experimental Diabetes: قم إيقف إعلاء العلف للأرلب لمــة

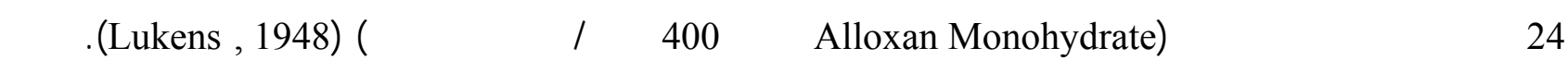

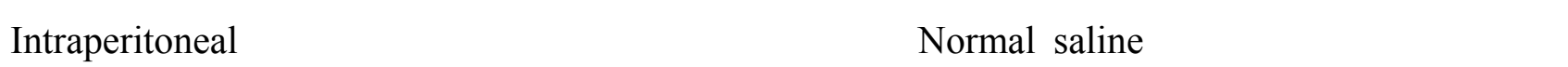

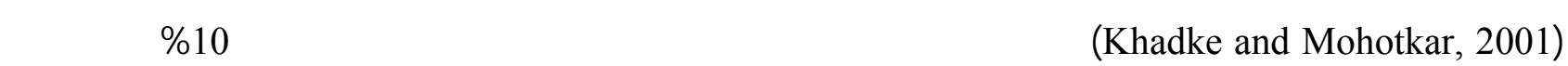
الكلوكوز لمدة 24ساعة وذك لفادي صنمة هبوط اللسكر نتيجة المعلملة بالألوكسان.

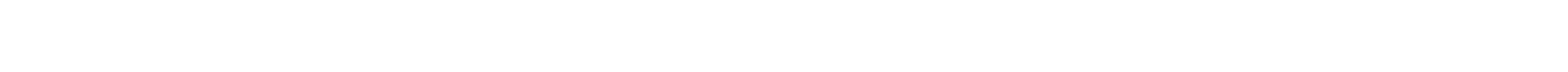

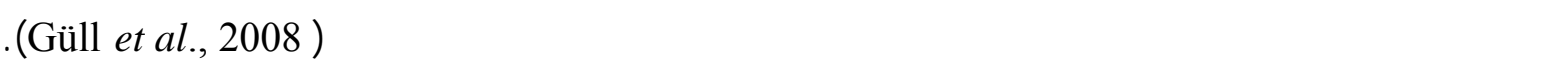

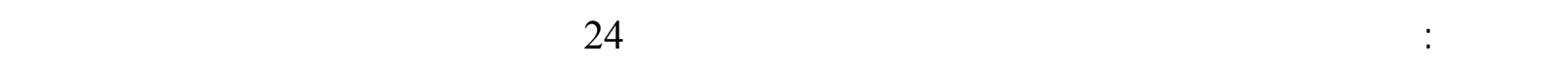

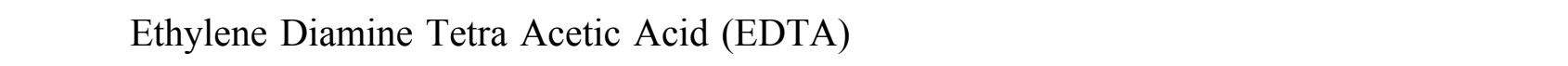

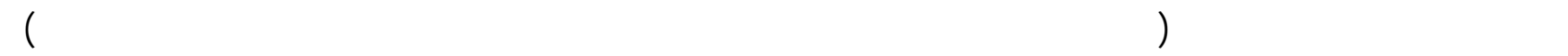

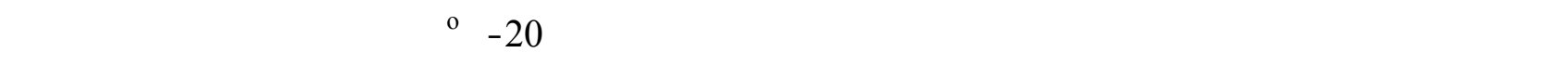
هرمون اللبتين. وقد لجريت فحوصل الدم بلستخدلم جهاز HeCo والمصنع من قلبشركة SEAC. أما مستوى هرمون اللبتين فقد ت م

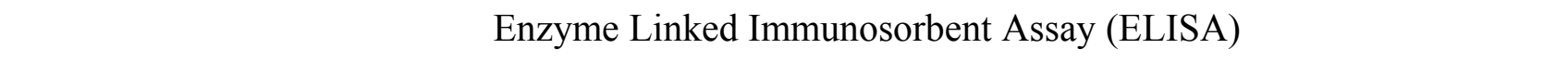

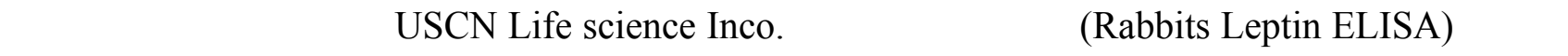
في Maffei ولخرون (1995). 


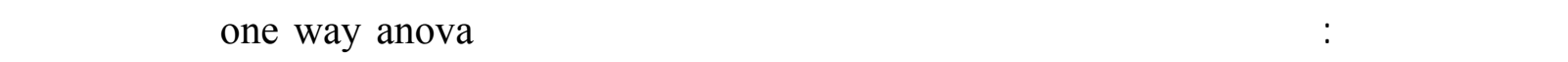

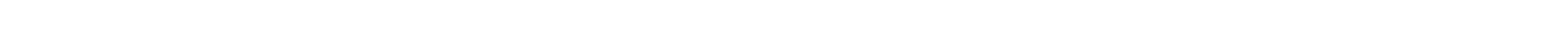
لحتمل (P > 0.05) وبلستخدم البرنلمج الإحصائي (2001) SAS لتحليل البيانت.

\section{النتائج والمنالشة}

تأثير مستخلص القرفة المغلي والألوكسلن في بعض مكونات الدم.

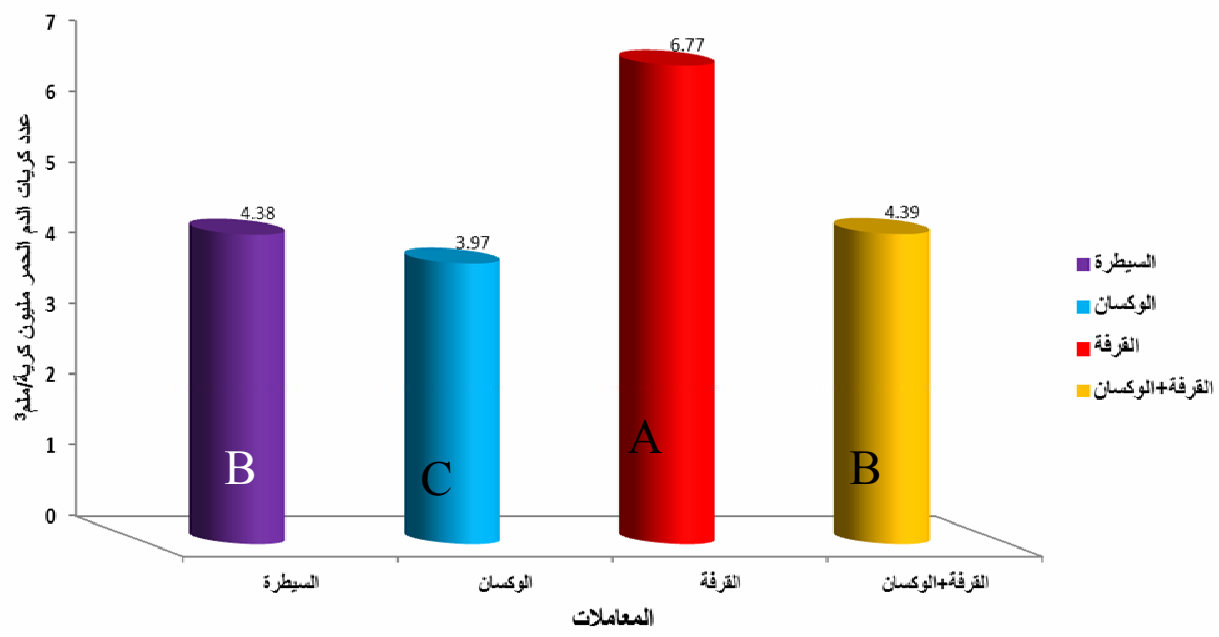

\section{المثل 1: تأثيرمستخلص الفرفة المغلي في عدكربك الم الحمرلإنث الأرلب النيوزلنية البيض.}

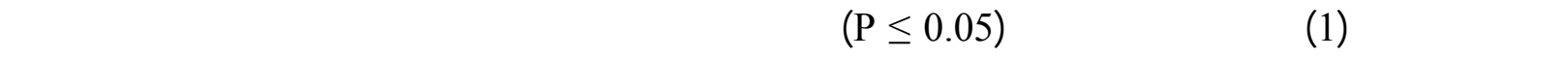

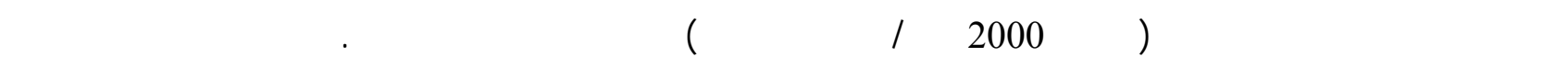

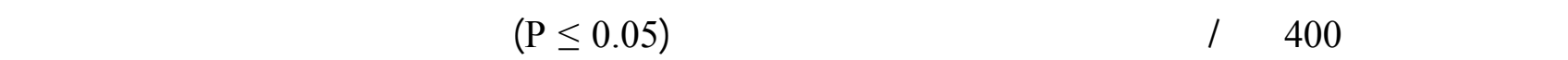

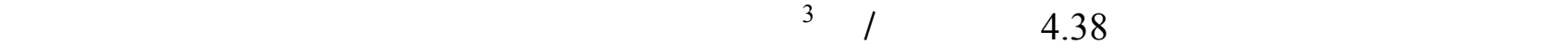

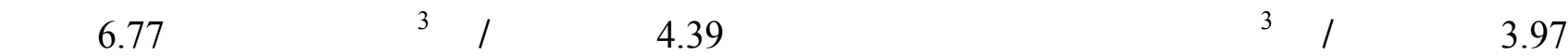
كرية /ملم 3

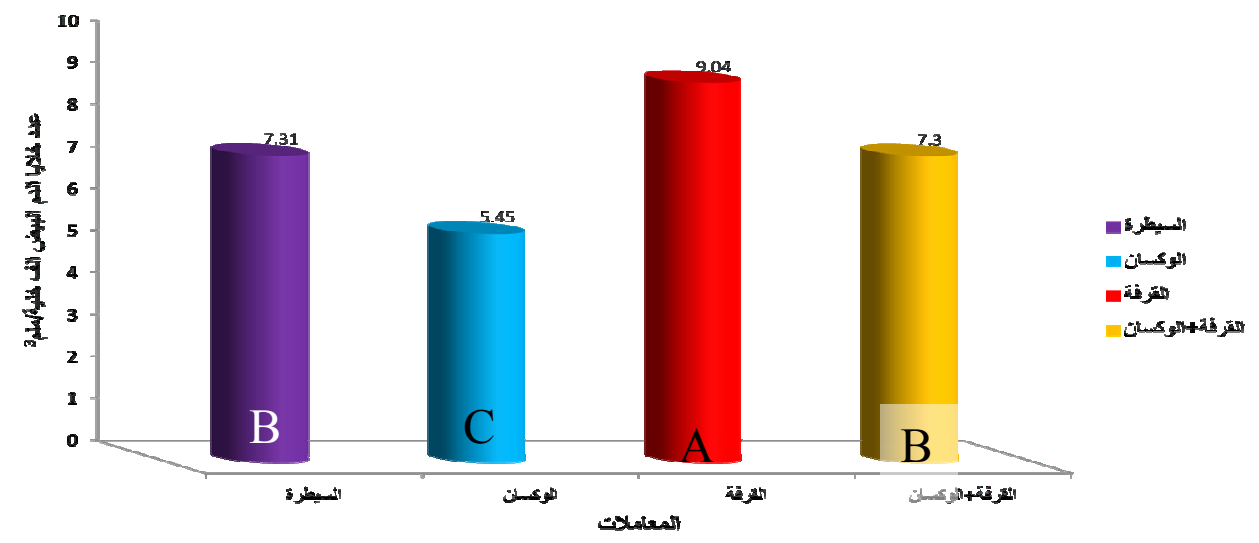


يتبين من الثكل (2) حدوث ارقفاع معنوي (P 1 (P) في عدد خلايا الدم البيض لإناث الأرالب النيوزلنية البيض

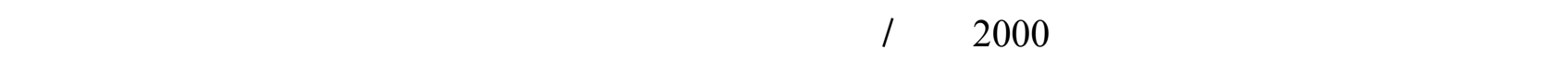

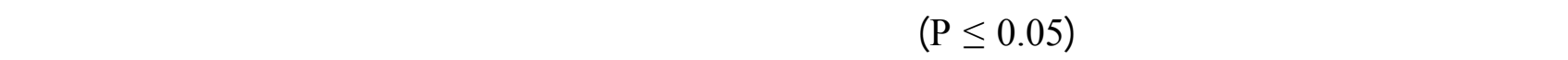
المسابي لمجوعة للسيطرة 7.31 أف خلية /ملم 3 ، بينما كلن المتوسط المسابي للمجموعة المعلملة بالقرفة 9.04 ألف خلي المة / 9.04 ملم ${ }^{3}$ المجموعة المعلملة بالألوكسان 5.45 ألف خلية /ملم 3 و المجموعة المعلملة بالقرفة مع الألوكسان 7.3 أل ف خلي لمة / .$^{3}$ مa

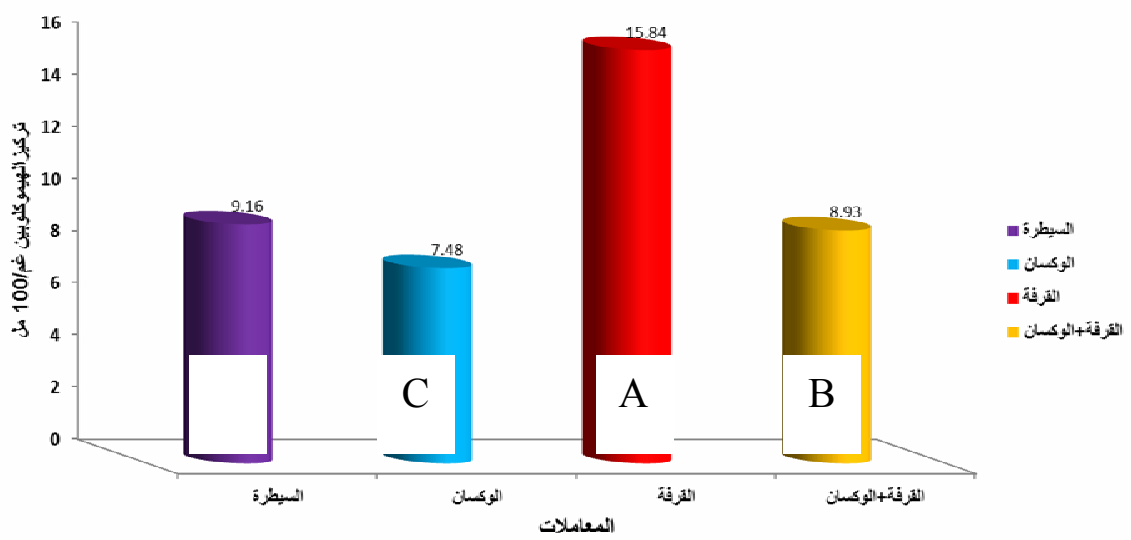

\section{الشكل 3: تأثير طستخاص الفرفة المغلي في تركيز الهيموكلوين لإنث الأرلب النيوزلنية البيض.}

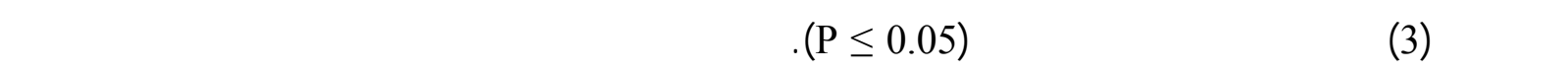

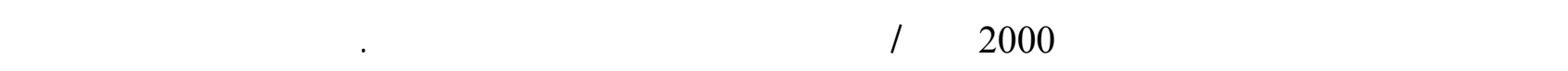

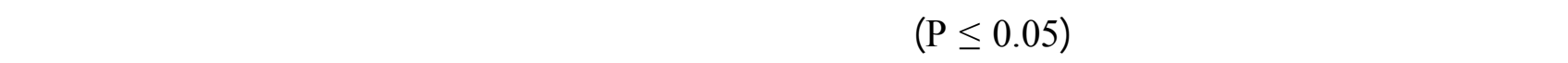

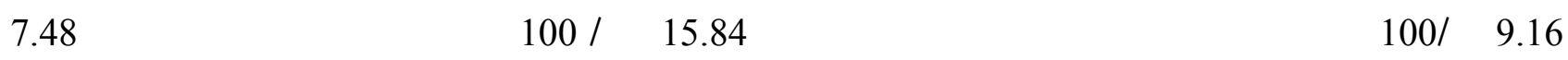

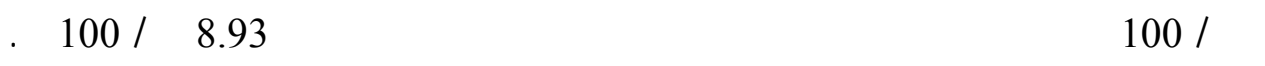

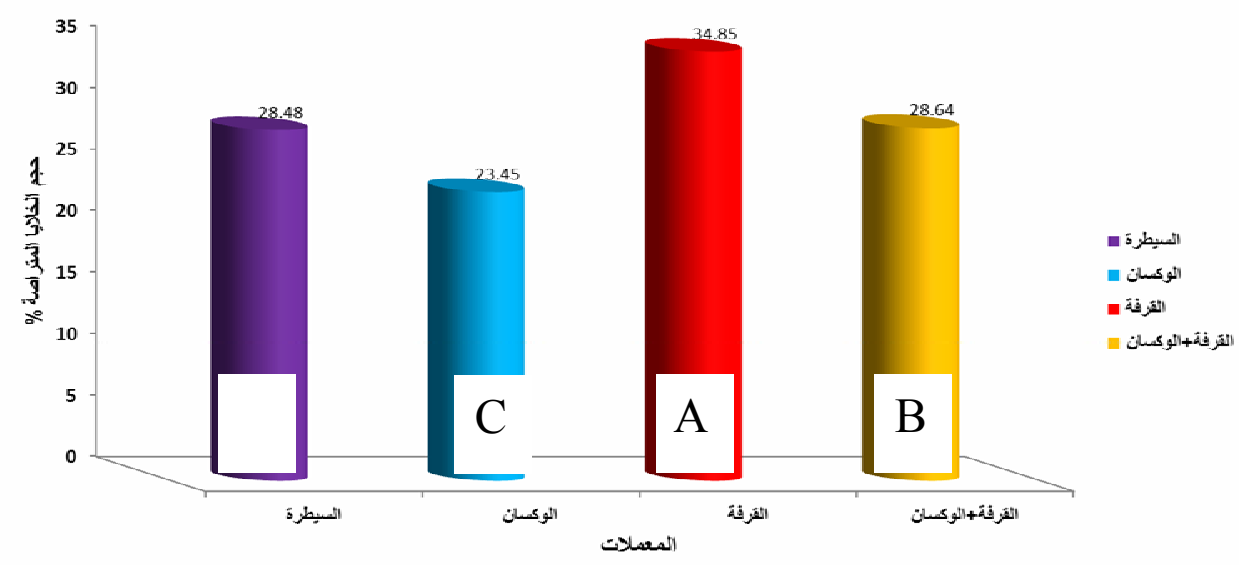


يوضح الثكل (4) حدوث ارتفاع معنوي في حهم خلايا الهم المرصوصة (P.C.V.) لإنث الأرافب النيوزلندية البيض

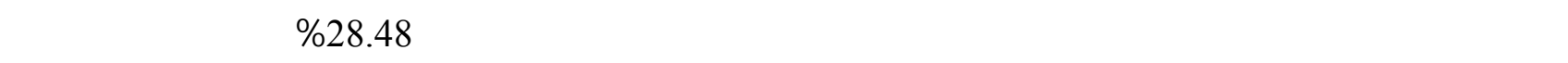

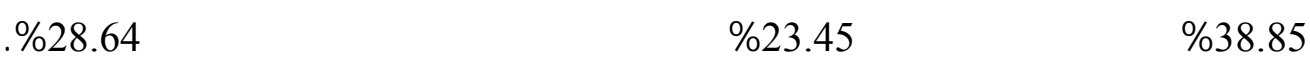

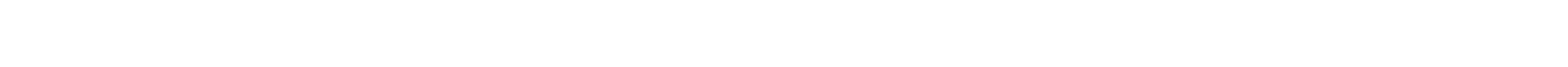

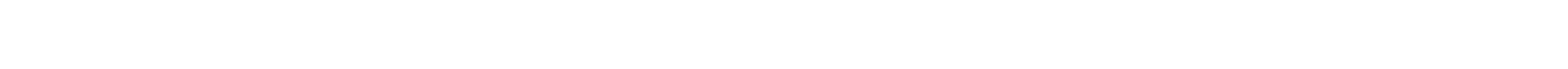

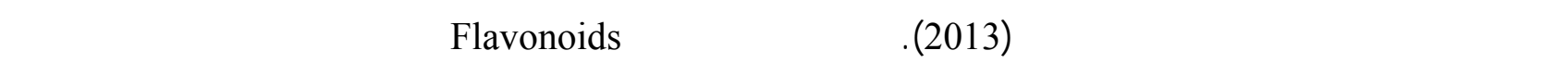

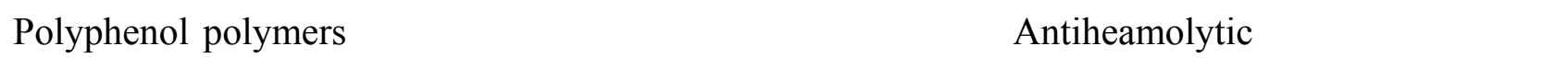
والكلايكوسيدات Glycosides التي تعد من أقوى مضادات الأكسة (للسيد، 2012) التي تمنع حصول الأك سرة ف في أغ شية الغية كريك الهم الحمر، بالإضافة إلى لحتواء اللحاء على عنصر الحيد الذي يساهم في زياة تكوين وإنتاج هيموكلوبين ال ـم ثة مم

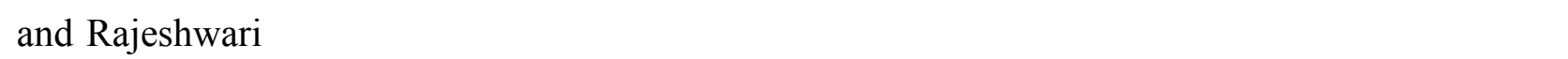
.(2011)Andallu

لماسبب حصول انغفاض معنوي في عدد كريت الدم الحمر في الأرافب المعلمل ـة بالألوك سلن مقارن سة مـ ع بـ القي المعلملات، فقد يعود إلى ارتفاع مستوى للسكر في الدم مما يسبب زيادة حصول تسكر Glycosylation لبروتين لت غ غ شاء

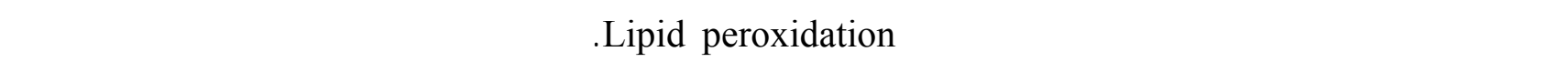
Hypoxia

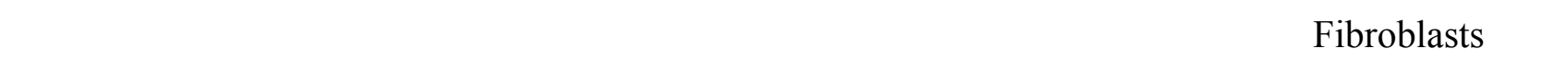

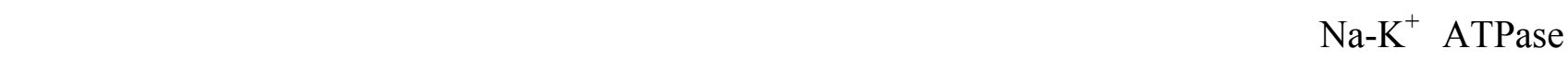
حدوث اضطررابلت في التبال للشعيري، ومن ثم تحل كريت الدم الحمر. كما إن انخفاض عدد كريلت الدم الحمر يرافقه أيضا

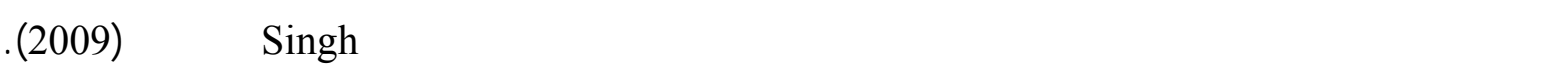

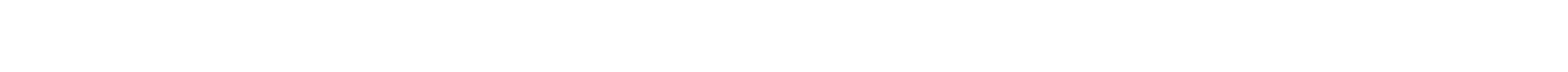

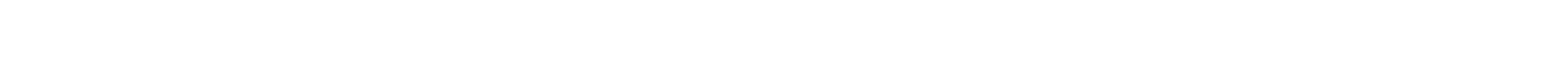
.(Sharma et al., 2011)

لماسبب الانخفاض المعنوي لإعداد خلايا الدم البيض في المجموعة المعلملة بالألوكسان مقارنة ببلقي المج لمبع, ف ان

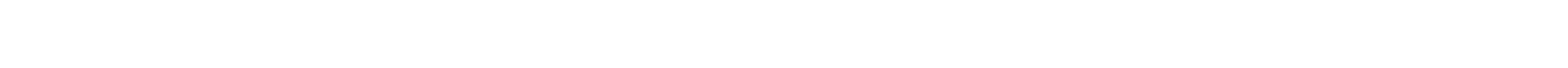

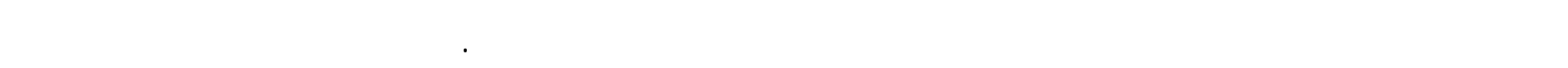

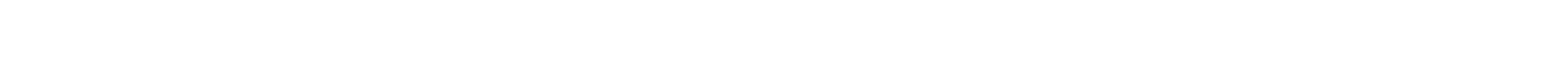
Seghrouchni 


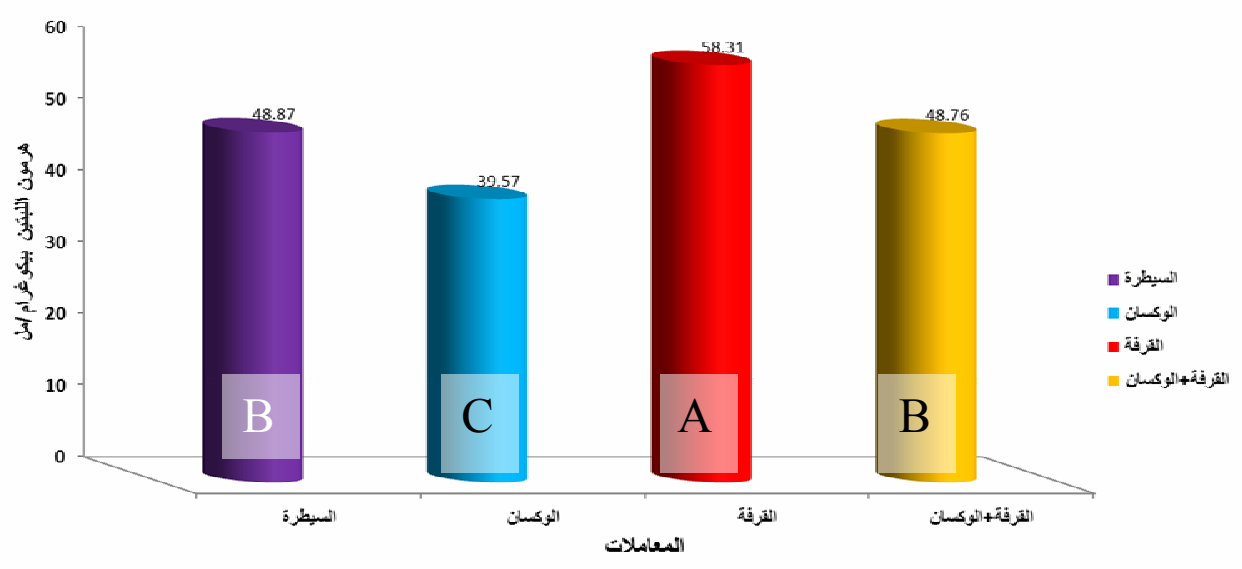

\section{اللثلى 5: تأثيرمستخلص الفرفة المغلي مستوى هرمهن اللبتن فمطل مم إنلث الأرلب النيوزلنية البيض.}

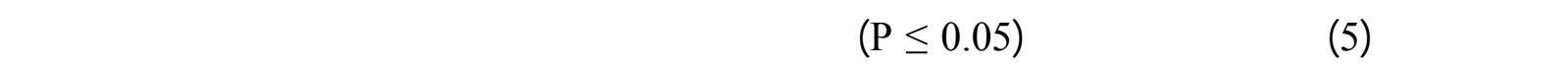

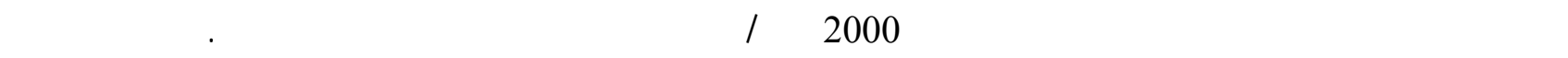

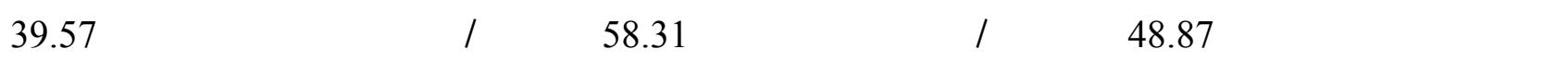

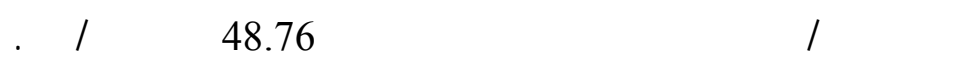

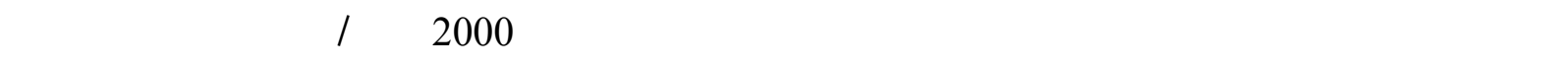

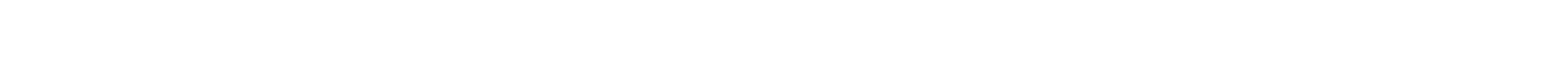

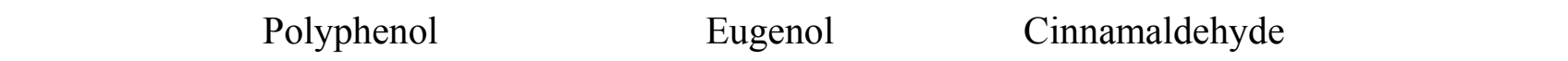

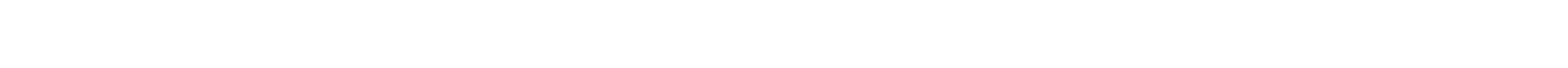

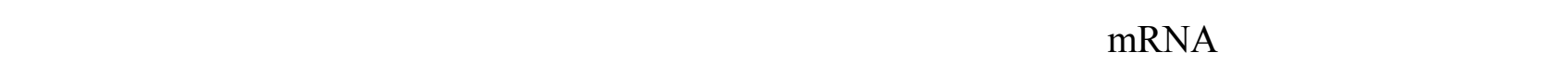
الكلوكوز (Steffan et al., 2001). أماسبب النخفاض مستوى هرمون اللبتين للمجموعة المعلملة بالألوكسان فربما يعود إلى زيادة لكسدة وحرق الـ دهون الإنت اجاج

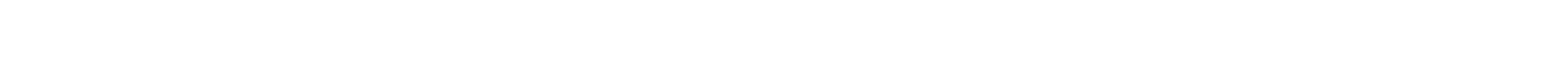
.(Miyanaga et al., 2003)

\section{المصار العربية المبية}

للسيد، عبد البلطط محمود (2012) ."موسوعةطب الأعثلب" المجلد الأول. الطبعة الثانية. الجيزة -مصر ص 61 - 61 -62.

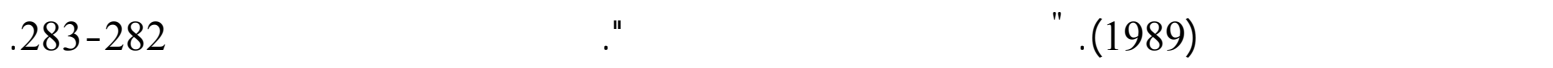

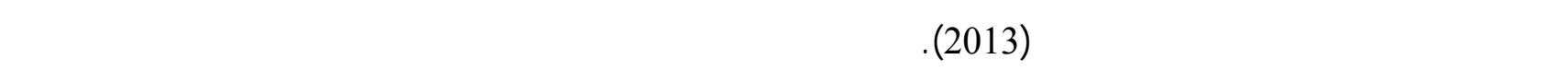

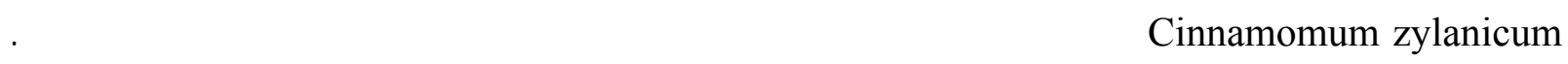
(مقبول للنشر) مجلة زراعة الرافين، جلمعة الموصل، الموصل، العراق.

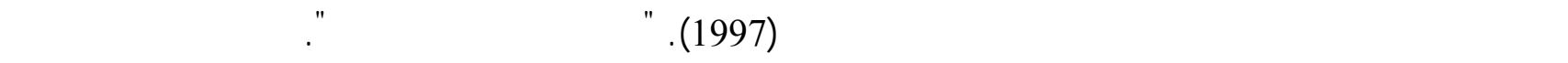

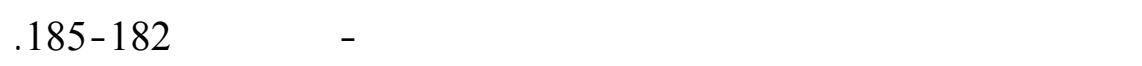

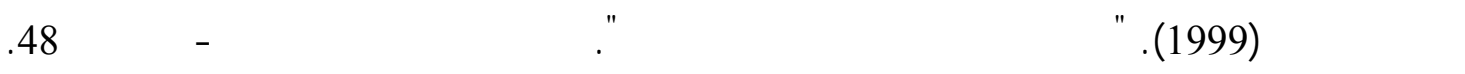




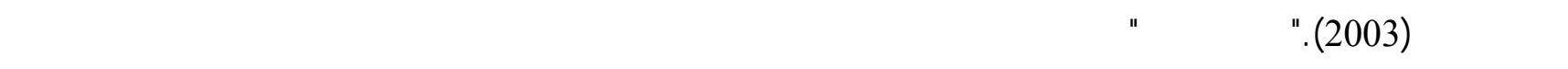

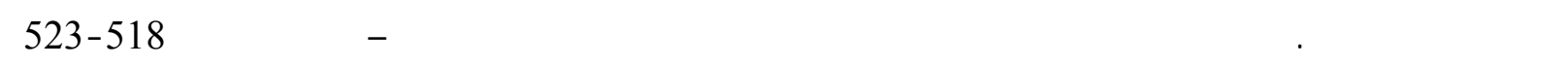
$.806-802$

Al-Rassam, Z.T.D. (2004). Urinary tract infection caused by Alcaligens spp. and its relation to immune suppression in diabetic patients.Ms.c. Thesis Mosul University. Mosul. Iraq.

Al-Rawi, S.; Fetters, M. (2012). Traditional Arabic and Islamic medicine: a conceptual model for clinicians and researchers. Glob. J. Heat. Sci. 4(3),164 -169.

Anderson, R.A.; Broadhurst, C.I.; Polansky, M.M.; Schmidt, W.F.; Khan, A.; Flanagon, V.P.; Schoene, N.W.; Graves, D.J. (2004). Isolation and characteration of polyphenol type-a polymers from cinnamon with insulin-like biological activity". J. Agric. Food Chem., 52, 6570.

Barnes, J.; Anderson, L.A.; Phillipson, J.D. (2002). "Herbal Medicines: A Guide for Healthcare Professionals". 2nd edn., Pharmaceutical Press, London, pp.135-136.

Chiej, R. (1984). " McDonald Encyclopedia of Medical Plants". McDonald and Co. (Puplishers) Ltd. Lond., pp. 26-208.

Engelgan, M.M. (2004). Diabetes diagnostic criteria and impaired glycemic states. Evol, evid.Clin. Diab. 22, 69-60.

Galleto, R.; Siqueira, V.L.; Ferreira, E.B.; Oliveira, A.J.; Bazotti, R.B. (2004). Absence of antidiabetic and hypolipidemic effect of Gymnema sylvester in non-diabetic and Alloxan diabetic rats. Bra. Arch. Bio. Tech. 47, 545-551.

Güll, N.; Cebesoy, S.; Özsoy, N. (2008). Lectins binding during Alloxan induced diabetes in rat soleus muscle. Afr. J. Biotechnol. 7(8), 926-930.

Khadke, V.V.; Mohotkar, A.L. (2001). Efficacy of prazosin, enalapril and amlodipine in Alloxan induced diabetic rabbits. Ind. MED. 98(3), 3-91.

Lukens, F.D.W. (1948). "Alloxan Diabetes".Cox Medical Research Institute. University of Pennsylvania. Philadelphia. 28, 303-330.

Maffei, M.; Halaas, J.; Raussin, E. (1995). Leptin levels in human and rodent: measurement of plasma Leptin and ob RNA in obese and weight reduced subjects. Nat. Med. 1(55), 55-61.

Miyanaga, F.; Ogawa, Y.; Ebihara, K.; Hidaka, S.; Tanaka, T.; Hayashi, I.; Masuza-ki, H.; Nakao, K. (2003). Leptin as an adjunct of Insulin therapy in Insulin deficient diabetes. Diabetologia. 46, 1329-1337.

Natural Resources Conservation Service (2004). "Plant Profile for Cinnamomum aromaticum (Cassia)". United States Department of Agricultural. New Yourk, Ny.USA. 481 p.

Pandit, N.N.; Singh, I.; Bhattacharjee, D.K.(1979). Impact of feeding chakwar (Casiatora) seed on the growth of broilers. Ind. J. Poul. Sci. 14,176.

Rajagopal, K.;Sasikala, K.(2008). Antihyperglycemic and antihyperlipidemic effects of Nymphaea stellata in Alloxan induced diabetic rats. Afri. J. Micbio. Res.49,137-141.

Rajeshwari, C.U.;Andallu, B.(2011). Oxidative stress in NIDDM patients: influence of Coriander (Coriandrum sativum) seeds. Res. J. Phar. 2(1), 31-41.

SAS (Statistical Analytical System) (2001). "SAS/STAT Users Guide For Personal Computers". Release. 6: 12.SAS Institute Inc., Cary, N.C., USA.

Seghrouchni, I.; Drai, J.; Bannier, E.; Riviere, J.; Calmard, P.; Garcia, I.; Orgiazzi, J.; Revol, A. (2002). Oxidative stress parameters in type I, type II and Insulin - treated type 2 diabetes mellitus; Insulin treatment efficiency. Clinica. Chimica. Acta. 321(1-2), 89-96. 
Sharma, V.; Kansal, L.; Sharma, A.; Lodi, S.; Sharma, S.H. (2011). Ameliorating effect of Coriandrum sativum extracts on hematological and immunological variables in an animal model of lead intoxication. J. Phar. Alli. Heal. Sci. 1(1), 16-29.

Singh, D.K.; Winocour, P.; Farrington, K. (2009). Erythropoietic stress and anemia in diabetes mellitus. Natu. Rev. Endo. 5, 204-210.

Steffan, N.; Fritsche, A.; Haring, H.; Stumvoll, M. (2001). Acute stimulation of Leptin concentration in humans during hyperglycemic hyperinsulinemia. Influence of free fatty acids and fasting. Int. J. Obes. Relat. Metab. Disord. 25(1), 138-142. 